\title{
Neurogenesis-Independent Antidepressant-Like Effects on Behavior and Stress Axis Response of a Dual Orexin Receptor Antagonist in a Rodent Model of Depression
}

\author{
Mathieu Nollet', Philippe Gaillard ${ }^{1,2}$, Arnaud Tanti', Virginie Girault', Catherine Belzung' \\ and Samuel Leman*, I \\ I'Inserm U930_-Imaging and Brain, Université François Rabelais, UFR Sciences et Techniques, Tours, France; ${ }^{2}$ Clinique Psychiatrique \\ Universitaire, CHRU de Tours, Tours, France
}

\begin{abstract}
Growing evidence indicates that an increase of orexin (or hypocretin) signaling is involved in the pathophysiology of major depression, but little is known regarding the causal link between the orexinergic system and depressive-like states. Here we blocked orexin receptors in mice subjected to unpredictable chronic mild stress (UCMS) to investigate putative antidepressant-like effects of this treatment, as well as the underlying mechanisms. BALB/c mice were exposed to 9 weeks of UCMS and from the third week onward treated daily with fluoxetine (20 mg/kg per day, per os) or with the dual orexin receptor antagonist almorexant ( $100 \mathrm{mg} / \mathrm{kg}$ per day, per os). The effects of UCMS regimen and pharmacological treatments were assessed by physical measures and behavioral testing. The dexamethasone suppression test was performed to examine the integrity of the negative feedback of the hypothalamic-pituitary-adrenal (HPA) axis, and immunohistochemical markers were used to assess cell proliferation (Ki-67), immature newborn neurons (doublecortin), and mature newborn neurons (5-bromo-2'-deoxyuridine/NeuN) in the dorsal and ventral parts of the hippocampus. Our results show that 7 weeks of fluoxetine or almorexant treatments counteract the UCMS-induced physical and behavioral alterations. Both treatments prevented the HPA axis dysregulation caused by UCMS, but only fluoxetine reversed the UCMS-induced decrease of hippocampal cell proliferation and neurogenesis, while chronic almorexant treatment decreased cell proliferation and neurogenesis specifically in the ventral hippocampus. Taken together, this is the first evidence that pharmacological blockade of the orexinergic system induces a robust antidepressant-like effect and the restoration of stress-related HPA axis defect independently from a neurogenic action.

Neuropsychopharmacology (2012) 37, 2210-2221; doi:I0.1038/npp.2012.70; published online 20 June 2012
\end{abstract}

Keywords: depression; orexin/hypocretin; antidepressant; dual orexin/hypocretin receptor antagonist; hippocampal neurogenesis; hypothalamic-pituitary-adrenal axis

\section{INTRODUCTION}

Major depression (MD), a pathology that can be triggered by chronic psychosocial stress in vulnerable subjects (El Hage et al, 2009; Keller et al, 2007), is characterized by mood disturbance, anhedonia, sleep abnormalities, weight changes, and dysregulation of the hypothalamic-pituitaryadrenal (HPA) axis (Nestler et al, 2002; Tanti and Belzung, 2010). Recently, adult hippocampal neurogenesis has been suggested either to participate in the etiology of MD (Snyder et al, 2011) or to be causally involved in the ability of antidepressant drugs to elicit a therapeutic response (Airan

*Correspondence: Dr S Leman, UMR Inserm 930_Imaging and Brain, Team 4: Affective Disorders, Université François Rabelais, UFR Sciences et Techniques, Parc Grandmont, 37200 Tours, France,

Tel: + 3302473669 97, Fax: + 3302473672 85,

E-mail: samuel.leman@univ-tours.fr

Received 30 January 2012; revised 16 March 2012; accepted 2 April 2012 et al, 2007; David et al, 2009; Jiang et al, 2005; Perera et al, 2011; Santarelli et al, 2003; Surget et al, 2008; Wang et al, 2008). Traditional antidepressants (e.g., selective serotonin reuptake inhibitors (SSRIs)) and novel drugs that exert antidepressant-like effects (e.g., antagonists at metabotropic glutamate receptors, synthetic cannabinoid HU210, tianeptine, compounds acting on the stress axis such as corticotropin-releasing hormone 1 (CRH1) or vasopressin V1b receptor antagonists, or melanin-concentrating hormone $(\mathrm{MCH})$ antagonist) both appear to increase hippocampal neurogenesis despite their different pharmacological mechanisms (Alonso et al, 2004; Czéh et al, 2001; David et al, 2007; Jiang et al 2005; McEwea et al, 2002; Yoshimizu and Chaki 2004). This was also observed with non-pharmacological therapy, such as electroconvulsive therapy (Malberg et al, 2000) or vagus nerve stimulation (Revesz et al, 2008). We have recently shown that this occurs via the potential of antidepressants to recruit new hippocampal neurons to rescue the HPA axis regulation defects (Surget et al, 2011), 
suggesting that restoration of the HPA axis feedback is sufficient to induce therapeutic effects, even in a context in which a given treatment does not stimulate hippocampal neurogenesis.

Orexins, also known as hypocretins, are a pair of excitatory neuropeptides called orexin-A (OX-A) and orexin-B (OX-B) (hypocretin-1 and hypocretin-2), synthesized from the common precursor prepro-orexin (de Lecea et al, 1998; Sakurai et al, 1998). They are produced by a population of neurons located in the dorsomedial hypothalamus (DMH), perifornical hypothalamic area (PFA), and lateral hypothalamus (LH), from which they send projections broadly all over the brain (Peyron et al, 1998). Physiological effects of orexins result from the activation of two G-protein-coupled receptors differentially distributed throughout the brain (Marcus et al, 2001; Trivedi et al, 1998), orexin receptor 1 and 2 (OXR1 and OXR2), with OX-A showing equal affinity for both receptors, while OX-B demonstrates a higher affinity for OXR2 (Sakurai et al, 1998).

Interestingly, orexins are implicated in several processes that are dysregulated in depressive subjects, including sleep/wake transitioning (Cao and Guilleminault, 2011; Sakurai et al, 2010), control of food intake (Cason et al, 2010; Thompson and Borgland, 2011), reward seeking (Aston-Jones et al, 2010; Harris et al, 2005), and HPA axis regulation (Lopez et al, 2010; Spinazzi et al, 2006), suggesting that orexins could be partly involved in the pathophysiology of MD. Nonetheless, preclinical and clinical studies that investigated the link between orexinergic system and MD still report conflicting results, showing that either hypoactivity (Allard et al, 2004; Brundin et al, 2007a; Ito et al, 2008; Lutter et al, 2008; Rotter et al, 2011) or hyperactivity (Feng et al, 2008; Mikrouli et al, 2011; Nollet et al, 2011; Salomon et al, 2003; von der Goltz et al, 2011) of the orexinergic system is associated with depressive state.

To further study the exact role of orexins in depressivelike states, we exposed mice to the unpredictable chronic mild stress (UCMS), a widely used naturalistic animal model of MD (Cryan and Holmes, 2005; Sibille et al, 2009; Surget et al, 2009; Willner, 1997). We then studied the behavioral and neurobiological effects of a chronic treatment with the dual orexin receptor antagonist almorexant (ALM) (Brisbare-Roch et al, 2007), compared to the classical antidepressant fluoxetine (FLX), a selective serotonin reuptake inhibitor (SSRI), with an emphasis on adult hippocampal neurogenesis and HPA stress axis. Here, we demonstrate that behavioral disturbances induced by chronic stress exposure are reversed by the dual orexin receptor antagonist, which counteracted HPA axis dysregulation through a neurogenesis-independent mechanism.

\section{MATERIALS AND METHODS}

\section{Animals}

In all, $95 \mathrm{BALB} / \mathrm{c}$ mice (Centre d'élevage Janvier, Le Genest St-Isle, France), aged between 6 and 7 weeks at the time of their arrival in the laboratory, were housed in groups of four per cage under standard conditions $\left(22 \pm 2{ }^{\circ} \mathrm{C}, 40 \%\right.$ humidity, inverted 12-h light-dark cycle with lights off at $0900 \mathrm{~h}$, food and water ad libitum) for 1 week before the beginning of experimental procedures. All experimental procedures were carried out in strict accordance with the European Communities Council directive (86/609/EEC) and received the approval of the ethical committee (agreement no. 2011-06-10, Comité d'Ethique en Expérimentation Animale Val de Loire, CEEAVdL).

\section{Experimental Design}

UCMS regimen used in this study has been previously presented in detail (Nollet et al, 2011) and is a variant of chronic mild stress procedures described by Willner (1997) in rats. Briefly, mice were subjected daily to various socioenvironmental low-intensity stressors according to an unpredictable schedule for a total period of 9 weeks (Figure 1). Pharmacological treatment started 2 weeks after the beginning of the UCMS protocol, and was always maintained until the end of the experiment. Body weight and coat state (high score indicates a coat in poor condition) were assessed weekly as markers of the progression of the UCMS-evoked syndrome. Behavioral

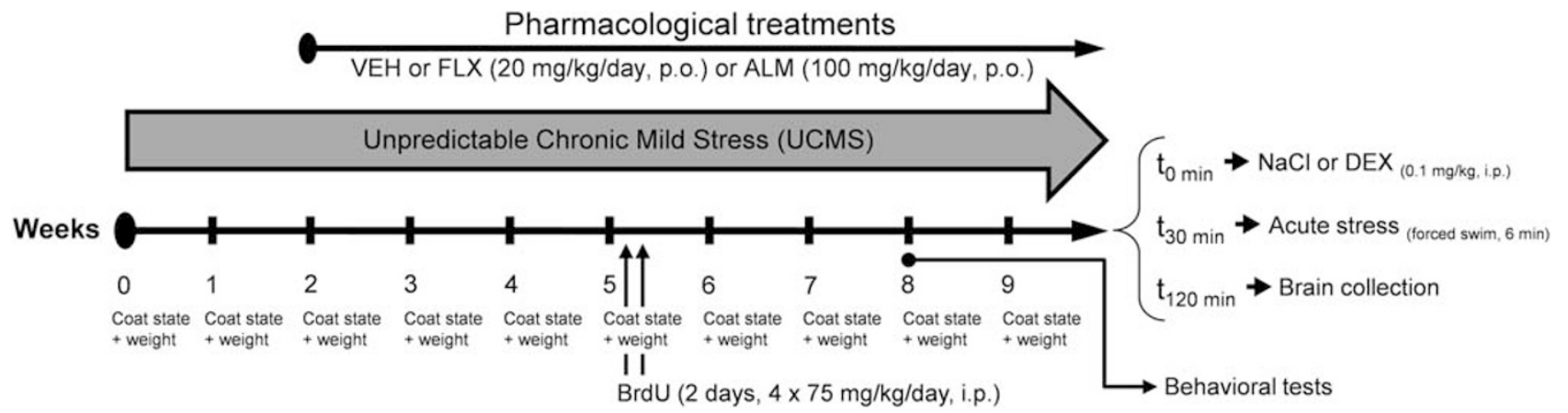

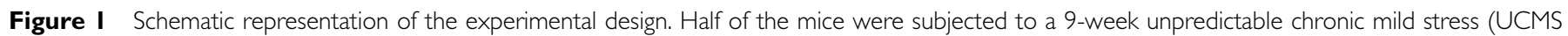

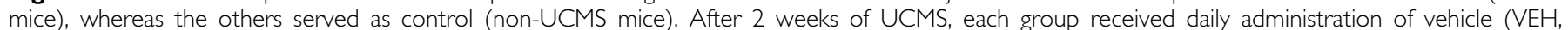

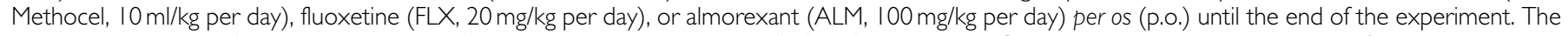

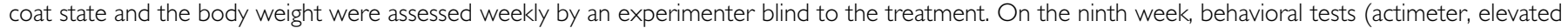

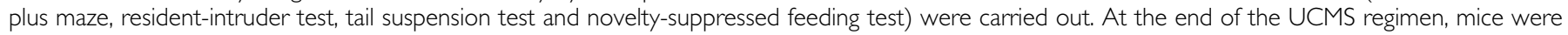

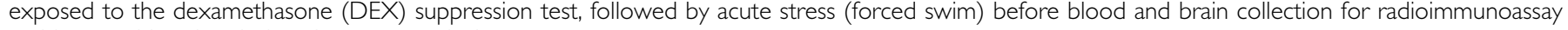
and immunohistochemical analyses, respectively. 
tests were performed in week 8 ( $n=14-19$ mice per group), at least $18 \mathrm{~h}$ after the last treatment (all mice were subjected to one test daily, always in the same sequence). To label newborn adult cells in the dentate gyrus (DG) of the hippocampus, the thymidine analogous 5-bromo- 2 '-deoxyuridine (BrdU, B5002; Sigma-Aldrich) was injected intraperitoneally 4 weeks before intracardiac perfusions (4 injections of $75 \mathrm{mg} / \mathrm{kg}$ every $2 \mathrm{~h}$, during 2 days). At the end of the UCMS protocol, all mice were subjected to dexamethasone (DEX) suppression test $2 \mathrm{~h}$ before being perfused. For further details, see Supplementary Materials and Methods.

\section{Drugs}

The SSRI FLX-HCl (Sequoia Research Products, Pangbourne, $\mathrm{UK}$ ) and the dual orexin receptor antagonist ALM (ACT078573-hydrochloride; Actelion Pharmaceuticals, Basel, Switzerland) were dissolved in $0.2 \%$ methylcellulose (Methocel, 64620; Sigma-Aldrich) water solution. Non-UCMS and UCMS mice daily received per os $(10 \mathrm{ml} / \mathrm{kg}$ per day) freshly prepared vehicle (VEH, $0.2 \%$ methylcellulose), FLX ( $20 \mathrm{mg} / \mathrm{kg}$ per day), or ALM ( $100 \mathrm{mg} / \mathrm{kg}$ per day). The selected dose for chronic ALM administration was based on previous experiment (Kang et al, 2009). Oral drug administrations were made between 1300 and $1500 \mathrm{~h}$, irrespective of the stress schedule.

\section{Behavioral Testing}

A brief description of the behavioral tests is presented here. For a full and detailed description, please refer to Supplementary Materials and Methods.

Basal locomotor activity. An actimeter was used $2 \mathrm{~h}$ (afternoon, ie, during the dark phase of the cycle), $10 \mathrm{~h}$ (night, ie, during the light phase of the cycle), and $18 \mathrm{~h}$ (morning, ie, at the beginning of the dark phase) after drug administration to assess the long-lasting effects of UCMS and pharmacological treatments on basal locomotor activity of mice in their home cage. Locomotor activity was measured during $2 \mathrm{~h}$ for each time point.

Elevated plus maze. The elevated plus maze (EPM) consists in a plus-cross-shaped maze, elevated from the floor, with two opposite enclosed arms and two others opened and brightly lit. Mice were placed in the center area facing one of the closed arms and their movements and time spent in the different arms were analyzed for $5 \mathrm{~min}$. The time spent in the open arms is associated with a reduction of anxiety behavior.

Resident-intruder test. The resident-intruder (R-I) test was performed as described previously (Nollet et al, 2011) and allows to measure the agonistic behavior of mice. The latency of the first attack and the number of attack(s) (data not shown) were measured over a 6-min period. Depressive-like animals are more agonistic and likely to attack more often and sooner than non-stressed animals (Mineur et al, 2003).

Tail suspension test. As described previously (Steru et al, 1985), mice were suspended by the tail using adhesive tape to a rod $60 \mathrm{~cm}$ above the floor. The trials were conducted for a period of $6 \mathrm{~min}$ and were video recorded. The behavioral measure was the duration of immobility, interpreted as behavioral despair.

Novelty-suppressed feeding test. The novelty-suppressed feeding (NSF) test used in this study has been described formerly (Surget et al, 2008). Fasted mice were placed in a corner of a square arena where a single pellet of food was placed on a white paper positioned in the box center. The latency to chew the pellet was recorded within a 3-min period. This test induces a conflicting motivation between the drive to eat the food pellet and the fear of venturing into the arena.

\section{DEX Suppression Test}

To assess the effects of UCMS and treatments on the HPA axis negative feedback, the glucocorticoid receptor agonist DEX-phosphate (D-1759; Sigma-Aldrich) was used to carry out a DEX suppression test. For this purpose, all mice were intraperitoneally injected with either DEX $(0.1 \mathrm{mg} / \mathrm{kg}$ in $0.9 \% \mathrm{NaCl}, n=5$ mice per group) or saline $(0.9 \% \mathrm{NaCl}, n=5$ mice per group). After $30 \mathrm{~min}$, mice were subjected to an acute stressor (forced swim) for $6 \mathrm{~min}$ to challenge the HPA axis in the same manner for all experimental groups whether they were chronically stressed or not. Then, 90 min later (ie, 120 min after DEX injection), mice were deeply anesthetized with sodium pentobarbital ( $40 \mathrm{mg} / \mathrm{kg}$, intraperitoneally), transcardially perfused, and their brains were collected for immunohistochemical analyses of Fos expression in the paraventricular nucleus (PVN). Blood was also collected for plasma corticosterone level analyses. Plasma was separated and stored at $-20^{\circ} \mathrm{C}$ until radioimmunoassay.

\section{Quantification of Corticosterone Levels}

Plasma was analyzed for total corticosterone levels using a ${ }^{125}$ I-labeled corticosterone double-antibody radioimmunoassay kit (MP Biomedicals, New York, NY) according to the manufacturer's protocol. To avoid inter-assay variability, all the samples were run in a single assay. Percentages of plasma corticosterone level's suppression induced by DEX injection were calculated taking the mean level of plasma corticosterone in animals of corresponding group injected with saline solution.

\section{Immunohistochemistry}

Immunohistochemical staining was performed to assess the neuronal activity (c-Fos protein) within the PVN of the hypothalamus (to assess the activity of this nucleus known to initiate the HPA axis cascade), the cell proliferation (Ki67 protein), and the neurogenesis (immature newborn neurons with doublecortin (DCX) staining and mature new neurons with BrdU $+\mathrm{NeuN}$ double staining) in the DG of hippocampus (Supplementary Figure 1; full immunohistochemical details are provided in Supplementary Materials and Methods). 


\section{Image Analyses and Cell Quantification}

Activity of hypothalamic nuclei related to HPA axis activity was assessed by the analysis of Fos-labeled neurons in the parvocellular nuclei of the PVN whose axons are known to release the $\mathrm{CRH}$. The nomenclature and nuclei boundaries used were those defined by Franklin and Paxinos's (2008) mouse brain atlas. Hippocampal cell proliferation and neurogenesis were assessed by the analysis of Ki-67-labeled (cell proliferation), DCX-labeled (newborn immature neu-
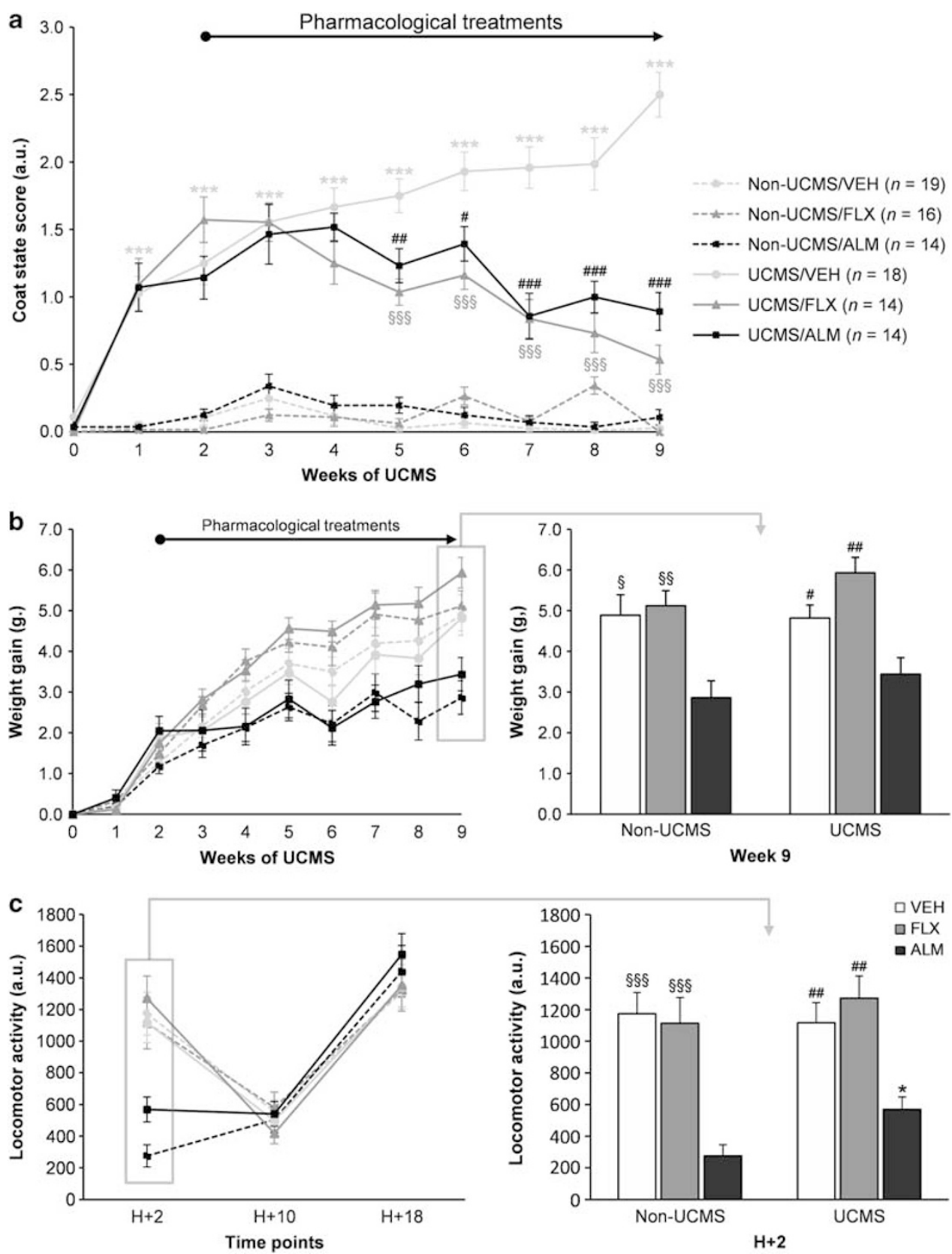

Figure 2 Effects of the unpredictable chronic mild stress (UCMS) and of 7-week treatment with fluoxetine (FLX, $20 \mathrm{mg} / \mathrm{kg}$ per day, per os (p.o.)) or almorexant (ALM, $100 \mathrm{mg} / \mathrm{kg}$ per day, p.o.) on the coat state, body weight and locomotor activity. (a) UCMS induced a significant deterioration of the coat state, as demonstrated by increasing coat state scores, from the week I until the end of the UCMS protocol (non-UCMS/vehicle (VEH) group vs *UCMS/ VEH group). Drug treatments initiated in the beginning of the third week of the UCMS exposure reversed this deterioration after 3 weeks of treatment (UCMS/VEH group vs ${ }^{\S}$ UCMS/FLX or ${ }^{\#}$ UCMS/ALM groups). No significant difference was observed between the non-UCMS groups. (b) A gradual increase of body weight was observed during the 9 weeks of UCMS without effects of chronic stress or FLX treatment, but with significant differences at the end of the experiment. ALM-treated mice presented slower weight gain in both non-UCMS mice (non-UCMS/ALM group vs ${ }^{\S}$ non-UCMS/VEH or ${ }^{\S}$ non-UCMS/FLX groups) and UCMS mice (UCMS/ALM group vs \#UCMS/VEH or \#UCMS/FLX groups). (c) ALM induced a decrease of locomotor activity only $2 \mathrm{~h}$ after administration in non-UCMS mice (non-UCMS/ALM group vs ${ }^{\S}$ non-UCMS/VEH or ${ }^{\S}$ non-UCMS/FLX groups) and in UCMS mice (UCMS/ALM group vs \#UCMS/VEH or \#CMS/FLX groups). In addition, this ALM-induced decrease of locomotor activity was greater in unstressed animals (non-UCMS/ALM vs *UCMS/ALM). Data represents mean \pm SEM; one symbol $p<0.05$, two symbols $p<0.01$, three symbols $p<0.001$; sample sizes indicated in (a) ( $n=14-19$ mice/group) concern all physical and behavioral measures illustrated in this Figure and in Figure 3. 
rons), and BrdU/NeuN-labeled (newborn mature neurons) cells in the granular cell layer of the DG. As dorsal and ventral parts of the hippocampus do not share the same connectivity with afferent and efferent areas (Fanselow and Dong, 2010), and as only the ventral part is known to regulate the HPA axis through polysynaptic neuronal circuit towards the PVN (Herman et al, 2005; Mizoguchi et al, 2003; Ulrich-Lai and Herman, 2009), cell proliferation and neurogenesis were examined separately in the dorsal, intermediate, and ventral parts of the hippocampus (Franklin and Paxinos, 2008). In this study, as results for dorsal and intermediate hippocampus are identical, only comparisons between dorsal and ventral parts are presented here (results for the intermediate part are presented in Supplementary Figure 2). Full explanation of image analyses and cell quantification are available in Supplementary Materials and Methods.

\section{Statistics}

Considering that relatively small sample sizes were used ( $n \leqslant 19$ mice per group for behavioral analysis and $n \leqslant 8$ mice per group for neurobiological analysis) and that assumptions for parametric statistics could not be ensured (normality and homoscedasticity), data were analyzed using the non-parametric Kruskal-Wallis 'ANOVA by ranks'
$H$-test. Significant effects (ie, $p<0.05$ ) were followed up with post-hoc Mann-Whitney $U$-tests with Holm-Bonferroni correction when appropriate (Aickin and Gensler, 1996). The Holm-Bonferroni method was not applied for DEX suppression test analysis due to the small sample size $(n=5$ mice per group). $p$-Values that are indicated in the Results section always derived from the between-groups comparisons using the Kruskal-Wallis $H$-test, while $p$-values resulting from post-hoc comparisons are indicated in the figures. All data are expressed as mean \pm standard error of the mean (SEM), and the sample sizes are provided in the figure legends.

\section{RESULTS}

\section{Physical and Behavioral Effects of UCMS and Pharmacological Treatments}

We first assessed the effects of chronic administration of ALM and FLX on physical state and behavior in mice subjected to 9-week UCMS or maintained under nonstressful conditions (non-UCMS mice).

UCMS induced a gradual deterioration of the coat state that appeared after 1 week of stress, whereas no degradation was observed in non-UCMS mice (Figure 2a). Interestingly, in contrast to UCMS/VEH mice whose physical state
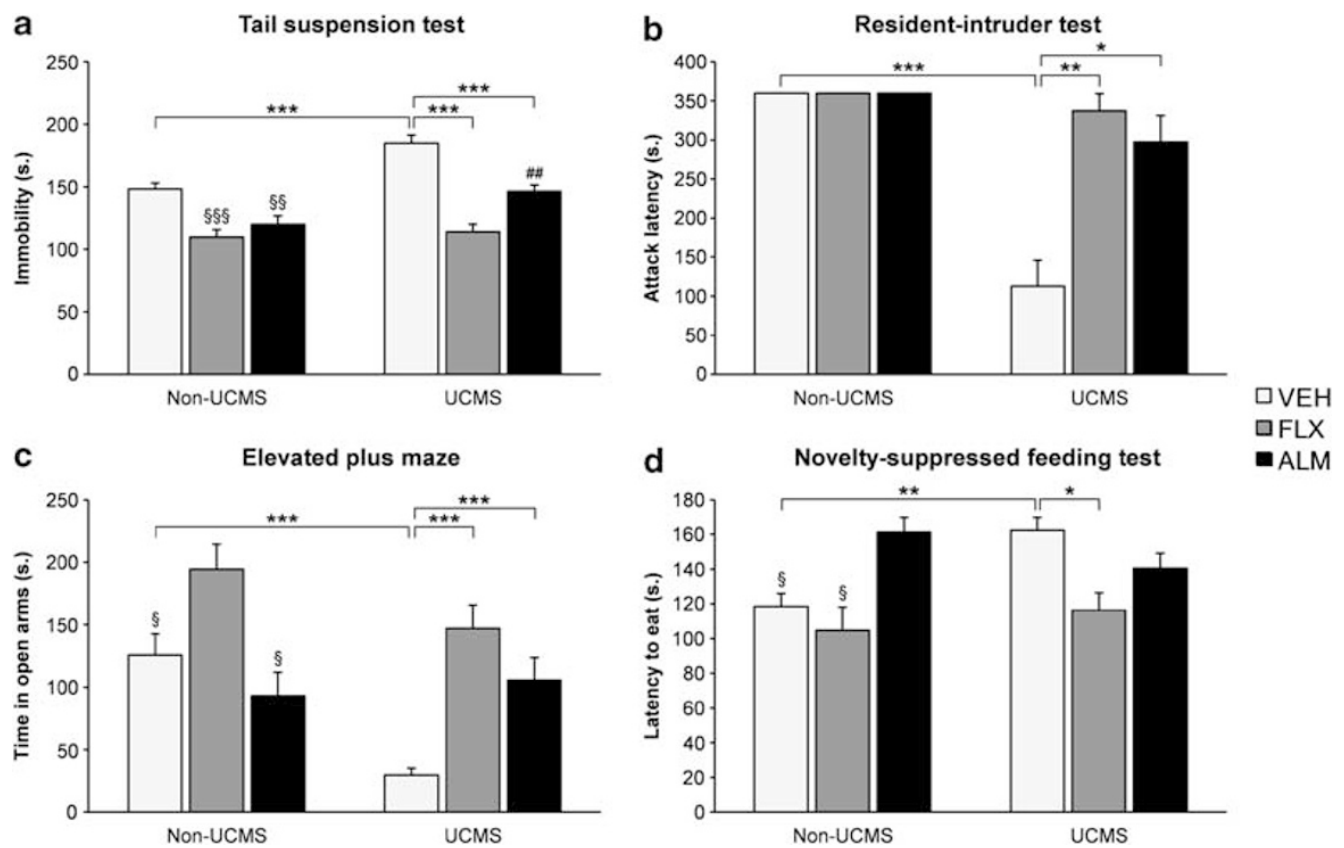

Figure 3 Effects of the unpredictable chronic mild stress (UCMS) and 7-week treatment with fluoxetine (FLX, $20 \mathrm{mg} / \mathrm{kg}$ per day, per os (p.o.)) or almorexant (ALM, $100 \mathrm{mg} / \mathrm{kg}$ per day, p.o.) on behaviors. (a) The UCMS increased the time of immobility in the tail suspension test (TST) (non-UCMS/ vehicle (VEH) group vs UCMS/VEH group), while pharmacological treatments decreased this alteration (UCMS/VEH group vs UCMS/FLX or UCMS/ALM groups). Pharmacological treatments also reduced the time of immobility in non-UCMS mice (non-UCMS/VEH group vs ${ }^{{ }^{8}}$ non-UCMS/FLX or ${ }^{\S}$ non-UCMS/ ALM groups). Furthermore, significant difference was also observed between the UCMS/FLX group and \#CMS/ALM groups. (b) The UCMS decreased the attack latency in the resident-intruder (R-I) test (non-UCMS/VEH group vs UCMS/VEH group), whereas pharmacological treatments reversed this agonistic behavior (UCMS/VEH group vs UCMS/FLX or UCMS/ALM groups). (c) In the elevated plus maze (EPM), the UCMS decreased the time spent in open arms (non-UCMS/NEH group vs UCMS/VEH group), whereas pharmacological treatments reversed this UCMS-induced effect (UCMS/VEH group vs UCMS/FLX

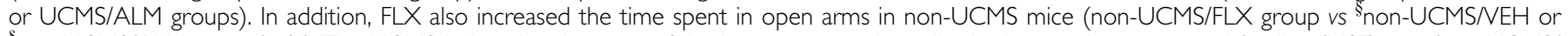

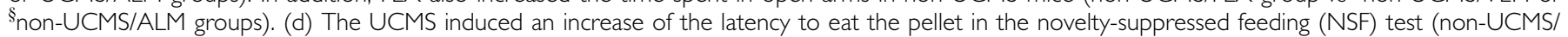
VEH group vs UCMS/VEH group), whereas FLX reversed this alteration (UCMS/VEH group vs UCMS/FLX group). However, ALM seemed to increase the latency to eat the pellet (non-UCMS/ALM group vs ${ }^{\S}$ non-UCMS/NEH or ${ }^{\S}$ non-UCMS/FLX groups). Data represents mean \pm SEM; $* p<0.05$, *** $<0.0$ I, **** $p<0.001 ; n=14-19$ mice per group as shown in Figure 2. 
worsened until the end of the UCMS procedure, chronic administration of FLX and ALM improved coat state reaching significance after 3 weeks of treatment (Figure 2a; full statistic details are provided in Supplementary Table 1). A gradual increase of body weight was observed during the 9 weeks of UCMS (Figure 2b). Although no effect of UCMS or chronic FLX treatment was noticed, chronic ALM administration significantly reduced the weight gain as it can be seen at the end of experimental protocol $(p<0.001$; Figure $2 b)$, without inducing any significant differences in body weight when compared with corresponding VEH groups (data not shown).

Locomotor activity was neither affected by UCMS or treatments $18 \mathrm{~h}$ after the last oral administration (at the time of the behavioral test), nor $10 \mathrm{~h}$ after (Figure 2c). Nevertheless, $2 \mathrm{~h}$ after administration of treatments, ALM significantly decreased the locomotor activity of mice in both UCMS and non-UCMS groups $(p<0.001$; Figure $2 c)$.

UCMS also induced behavioral alterations that were consistently restored after pharmacological treatments (except in the NSF test for ALM-treated mice). Importantly, none of these alterations were due to changes in locomotor activity, as all behavioral tests were carried out at a time point at which no change in locomotion was observed. In the TST, UCMS induced an increase of immobility, an effect counteracted by FLX and ALM treatments in both UCMS and non-UCMS mice ( $p<0.001$; Figure 3a). Furthermore, in the R-I test, a decrease of attack latency was observed in UCMS/VEH mice, reversed by FLX and ALM treatments $(p<0.001$; Figure 3b). No effect of treatments was observed in non-UCMS mice. Moreover, in the EPM, UCMS reduced the time spent in open arms, an effect reversed by FLX and
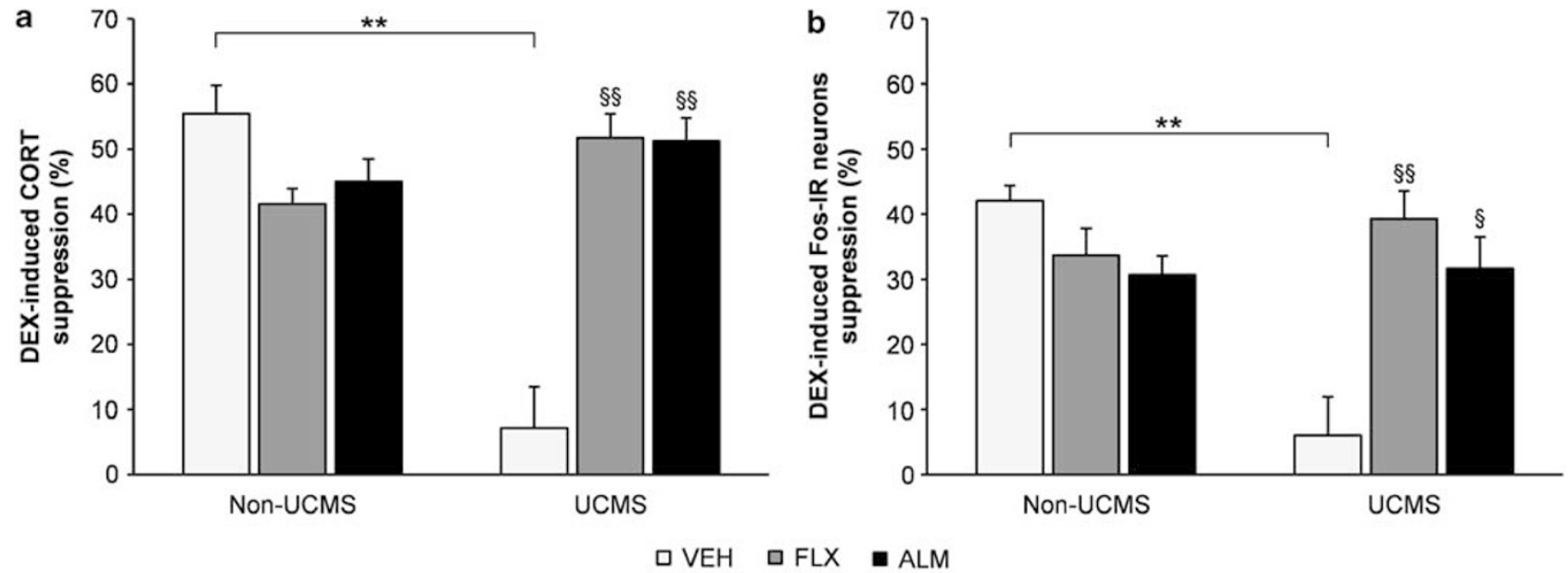

C

Non-UCMS/VEH

UCMS/VEH
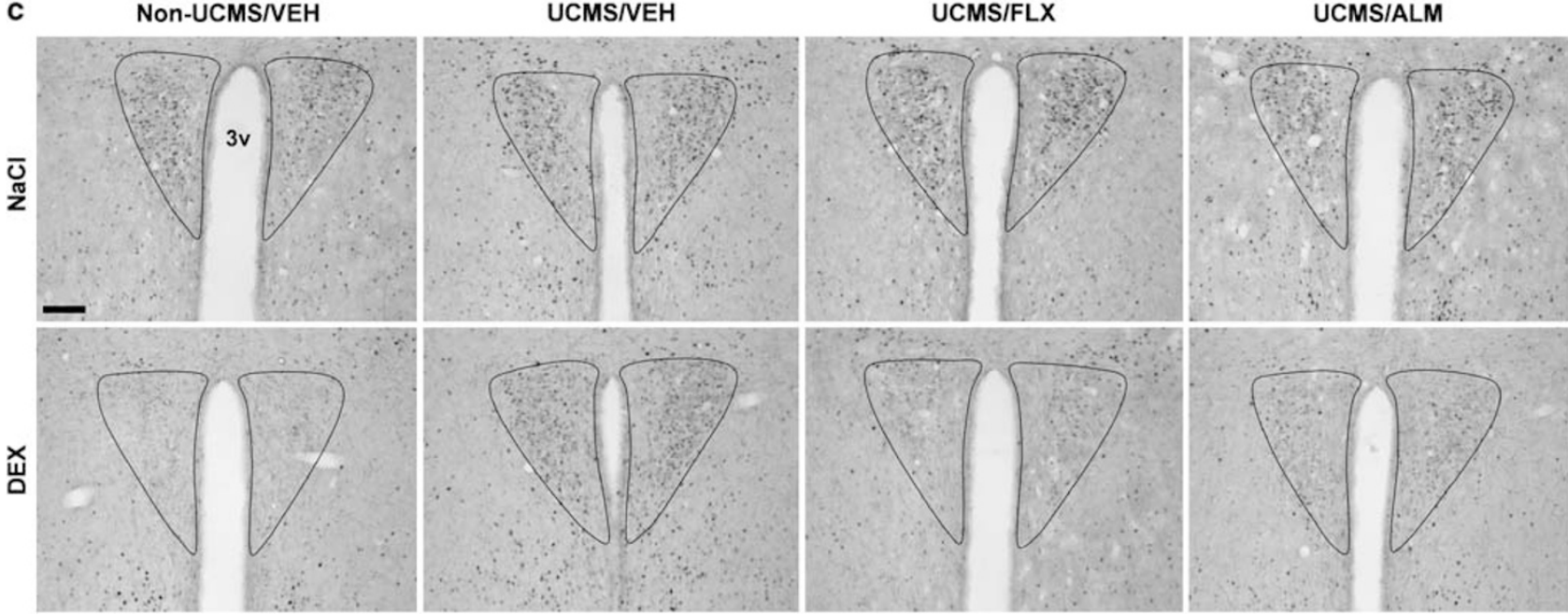

Figure 4 Effects of the unpredictable chronic mild stress (UCMS) and 7-week treatment with fluoxetine (FLX, $20 \mathrm{mg} / \mathrm{kg}$ per day, per os (p.o.)) or almorexant (ALM, $100 \mathrm{mg} / \mathrm{kg}$ per day, p.o.) on the dexamethasone (DEX) suppression test. (a) The UCMS induced a decrease of the DEX-induced suppression of plasma corticosterone (CORT) (non-UCMS/vehicle (VEH) group vs UCMS/VEH group), whereas FLX and ALM treatments reversed this alteration (UCMS/VEH group vs ${ }^{\S}$ UCMS/FLX or ${ }^{\S}$ UCMS/ALM groups). (b) The UCMS also decreased the DEX-induced suppression of Fos-immunoreactive neurons in the parvocellular nuclei of the paraventricular nucleus (PVN) (non-UCMS/NEH group vs UCMS/VEH group), whereas both pharmacological treatments counteracted this alteration (UCMS/VEH group vs ${ }^{\S}$ UCMS/FLX or ${ }^{\S} U C M S / A L M$ groups). (c) Representative images of single-labeled Fosimmunoreactive neurons (in black) counted in the parvocellular nuclei of the PVN, around the third ventricle (3v), to assess the reactivity of the negative feedback of the hypothalamic-pituitary-adrenal (HPA) axis through the DEX suppression test (magnification bar, I $00 \mu \mathrm{m}$ ). In graphs, I00\% value represents a full DEX-induced suppression of plasma corticosterone or Fos expression in the PVN. Data represent mean \pm SEM; one symbol $p<0.05$, two symbols $p<0.01 ; n=5$ mice per group. 
ALM treatments, while FLX also increased the time spent in open arms in non-UCMS mice $(p<0.001$; Figure $3 c)$. Finally, in the NSF test, UCMS increased the latency to eat, an effect restored by FLX ( $p<0.001$; Figure $3 \mathrm{~d})$. No effect of FLX was observed in non-UCMS mice. Nevertheless, in the NSF test, ALM did not block the behavioral alteration induced by UCMS, as this treatment significantly increases the latency to eat the pellet in non-UCMS group. However, when returned to their home cage, mice did not display any difference in the latency to smell the pellet or in the food consumption 5 min after the test (data not shown).

\section{Effects of UCMS and Pharmacological Treatments on HPA Axis Negative Feedback}

Depressive states are often associated with hyperactivity of HPA axis that can be reversed by antidepressants (Ising et $a l, 2007)$. We thus examined the integrity of the negative feedback of the HPA axis through the DEX suppression test following UCMS and chronic pharmacological treatments. In this test, the administration of the glucocorticoid receptor agonist DEX-phosphate $(0.1 \mathrm{mg} / \mathrm{kg}$, intraperitoneally) is known to decrease the plasma corticosterone level and to attenuate or suppress the activation of the PVN parvocellular nuclei through the activation of the negative feedback when it is undamaged.

We found that UCMS dampened the effectiveness of the negative feedback loop of the HPA axis and this was reversed by the pharmacological treatments. Indeed, the percentage of corticosterone suppression induced by DEX injection was strikingly decreased by UCMS (non-UCMS/ $\mathrm{VEH}$ mice $\sim 55 \%$ vs UCMS/VEH mice $\sim 7 \%$ ), and this effect was reversed after chronic FLX $(\sim 52 \%)$ and ALM $(\sim 51 \%)$ treatments $(p<0.01$; Figure $4 \mathrm{a})$. The percentage of Fos-positive neurons in the PVN was also reduced following
UCMS (non-UCMS/VEH mice $\sim 42 \%$ vs UCMS/VEH mice $\sim 6 \%)$, whereas FLX $(\sim 39 \%)$ and ALM $(\sim 32 \%)$ treatments restored the Fos activation of PVN neurons $(p<0.01$; Figure 4b). Importantly, no significant effect of UCMS or treatments on plasma corticosterone level or Fos expression in the parvocellular nuclei of the PVN in control mice receiving saline solution was noticed (data not shown).

\section{Effects of UCMS and Pharmacological Treatments on Hippocampal Cell Proliferation, Neurogenesis, and Neuronal Survival}

Antidepressant drugs require or modulate hippocampal cell proliferation or neurogenesis (Santarelli et al, 2003; Surget et al, 2008, 2011). We therefore studied the ability of chronically administrated ALM and FLX to modulate hippocampal cell proliferation and neurogenesis in UCMSand non-UCMS-subjected mice.

For this purpose, we first assessed UCMS and treatment effects on hippocampal cell proliferation using Ki-67 marker. We observed that UCMS induced a decrease of Ki-67-positive cell density in the dorsal hippocampus ( $\sim-46 \%$, nonUCMS/VEH vs UCMS/VEH, $p<0.001$; Figure 5a), and a greater decrease in the ventral part of hippocampus ( -63\%, non-UCMS/VEH vs UCMS/VEH, $p<0.001$; Figure $5 \mathrm{a})$. These reductions were reversed by FLX in both parts of the DG. UCMS/FLX mice exhibited a significantly higher Ki-67-positive cell density than in non-UCMS/FLX group, with no effect of FLX in the non-UCMS group, suggesting that FLX increased cell proliferation only in stressed mice. Interestingly, Ki-67-positive cell density was not increased in either parts of the hippocampus following chronic ALM in UCMS mice and was decreased in the ventral hippocampus of non-UCMS animals following chronic ALM treatment.

Figure 5 Effects of the unpredictable chronic mild stress (UCMS) and 7-week treatment with fluoxetine (FLX, $20 \mathrm{mg} / \mathrm{kg}$ per day, per os (p.o.)) or almorexant (ALM, $100 \mathrm{mg} / \mathrm{kg}$ per day, p.o.) on the cell proliferation, neurogenesis and neuronal survival in the dorsal and the ventral hippocampus. (a) The cell proliferation was assessed by the number of Ki-67-positive cells per $\mathrm{mm}^{3}$ of the granule cell layer (GCL). In the dorsal hippocampus, the UCMS induced a significant decrease of cell proliferation (Non-UCMS/vehicle (VEH) group vs UCMS/VEH group), reversed by FLX (UCMS/VEH vs UCMS/FLX group) but not by ALM (UCMS/FLX group vs UCMS/ALM group). Significant differences were also observed between the non-UCMS/FLX group vs ${ }^{\S} \cup C M S / F L X$ group, and between the non-UCMS/ALM group and "UCMS/ALM group). In the ventral hippocampus, the UCMS also decreased the cell proliferation (nonUCMS/NEH group vs UCMS/NEH groups), whereas FLX treatment reversed this alteration (UCMS/VEH group vs UCMS/FLX groups) without any effect of

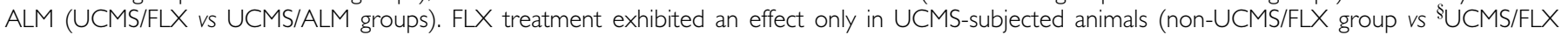
groups). Furthermore, ALM treatment reduced the ventral hippocampal cell proliferation (non-UCMS/VEH or non-UCMS/FLX groups vs non-UCMS/ALM group). (b) The generation of immature neurons was assessed by the number of doublecortin (DCX)-positive cells per $\mathrm{mm}^{3}$ of the granule cell layer (GCL). In the dorsal part of hippocampus, the UCMS induced a decrease of immature neurons genesis (non-UCMS/VEH group vs UCMS/VEH group), while treatment with FLX counteracted this reduction (UCMS/VEH group vs UCMS/FLX group). No effect of ALM was noticed in stressed mice (UCMS/FLX group vs UCMS/ALM group). A significant difference was also observed between the non-UCMS/ALM group and ${ }^{\circledR} U C M S / A L M$ group. In the ventral hippocampus, the UCMS decreased the immature neurons immunoreactivity (non-UCMS/VEH group vs UCMS/VEH group), which was reversed by FLX (UCMS/VEH group vs UCMS/FLX). Concerning ALM treatment, in addition to the absence of treatment effect in the UCMS group (UCMS/FLX group vs UCMS/ALM group), the orexinergic antagonist decreased ventral hippocampal neurogenesis in non-UCMS mice (non-UCMS/VEH group or non-UCMS/ FLX group vs non-UCMS/ALM group). (c) The density of mature neurons (4 weeks old) was assessed by the number of NeuN-positive cells expressing 5-bromo-2'-deoxyuridine (BrdU) marker per $\mathrm{mm}^{3}$ in the (GCL). In the dorsal hippocampus, the UCMS decreased the density of mature neurons (nonUCMS/NEH group vs UCMS/VEH group), whereas FLX treatment reversed this alteration (UCMS/VEH group vs UCMS/FLX group). Although no effect of ALM was observed (UCMS/FLX group vs UCMS/ALM group), FLX increased the proportion of mature neurons in non-UCMS mice (non-UCMS/FLX group vs non-UCMS/NEH or non-UCMS/ALM groups, and non-UCMS/FLX group vs ${ }^{\S} U C M S / F L X$ group). A significant difference was also observed between nonUCMS/ALM group and ${ }^{\#}$ UCMS/ALM group. In the ventral part of hippocampus, a UCMS-induced decrease of the density of mature neurons was noticed (non-UCMS/NEH group vs UCMS/NEH group), reversed by FLX (UCMS/VEH group vs UCMS/FLX group) but not by ALM (UCMS/FLX group vs UCMS/ ALM group). Furthermore, the treatment with ALM decreased the neurogenesis in the ventral hippocampus (non-UCMS/VEH group vS non-UCMS/ALM group), where as the UCMS further reduced the number of mature neurons (non-UCMS/ALM group vs \#UCMS/ALM group). FLX treatment also increased neurogenesis in non-UCMS mice (non-UCMS/FLX group vs non-UCMS/VEH or non-UCMS/ALM groups, and non-UCMS/FLX group vs ${ }^{\S} U C M S / F L X$ group. Data represent mean \pm SEM; $* p<0.05$, $* * * 0.01 ; n=8$ mice/group. 
We then focused on the newborn immature neurons by assessing the density of DCX-expressing cells in the DG. We found that UCMS decreased hippocampal DCX-positive cell density in the dorsal hippocampus ( $\sim-35 \%$, non-UCMS/ VEH $v s$ UCMS/VEH, $p<0.001$; Figure $5 \mathrm{~b}$ ), and even more in the ventral hippocampus ( $\sim-56 \%$, non-UCMS/VEH $v s$ UCMS/VEH, $p<0.001$; Figure 5b). FLX treatment significantly reversed this disruption, whereas no change of DCXpositive cell density was seen in ALM-treated UCMS mice in both parts of the hippocampus. Furthermore, in the ventral hippocampus, non-UCMS mice chronically treated with ALM exhibited a decrease of DCX-positive cell density.

Finally, we examined the survival of adult newborn neurons through double-labeled cells with the neuronal marker NeuN and the cellular division marker BrdU (injected 4 weeks before animals scarifice). We observed a UCMS- mediated decrease in hippocampal BrdU/NeuN-positive cell density in the dorsal ( $\sim-73 \%$, non-UCMS/VEH $v s$ UCMS/ $\mathrm{VEH}, p<0.001$; Figure $5 \mathrm{c}$ ) and particularly in the ventral part of hippocampus ( $-86 \%$, non-UCMS/VEH $v s$ UCMS/VEH, $p<0.001$; Figure $5 c$ ), an effect again reversed by FLX, but not by ALM. The latter also decreased the density of BrdU/NeuNpositive neurons in the ventral hippocampus in non-UCMS mice. In addition, we found that in non-UCMS mice, FLX significantly increased the proportion of mature neurons in dorsal and ventral hippocampus compared with other nonstressed animals.

To summarize, chronic ALM treatment was unable to counteract the UCMS effects on cell proliferation, neurogenesis, and neuronal survival in the entire DG, and even decreased it in the ventral hippocampus of non-stressed animals. a

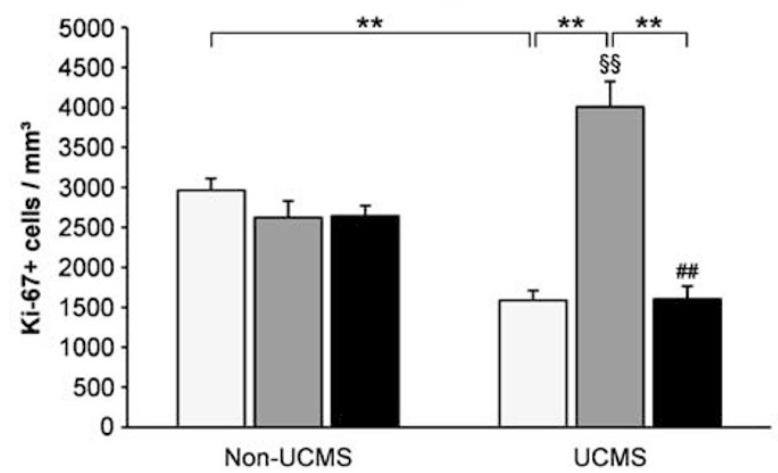

b

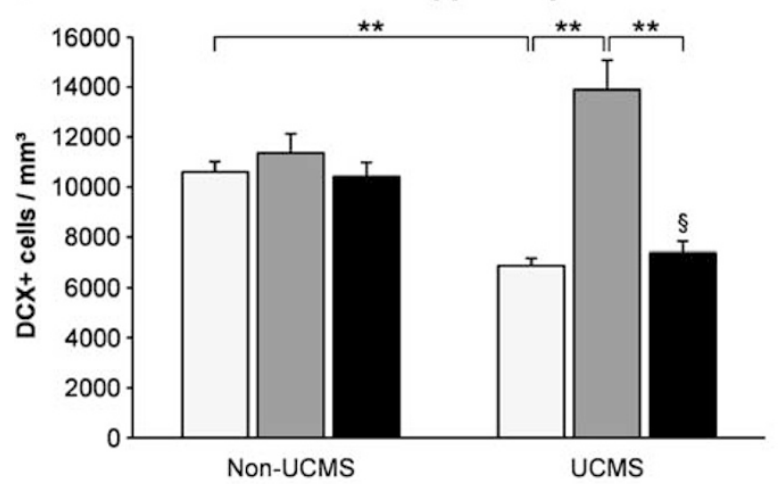

C

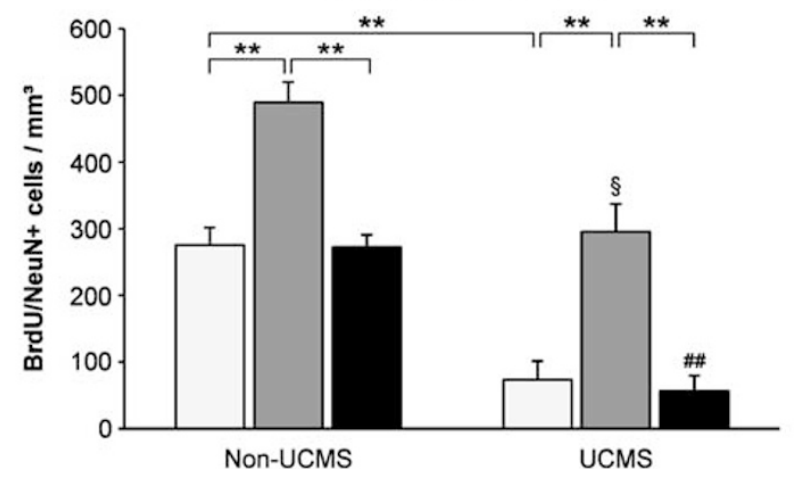

Ventral hippocampus

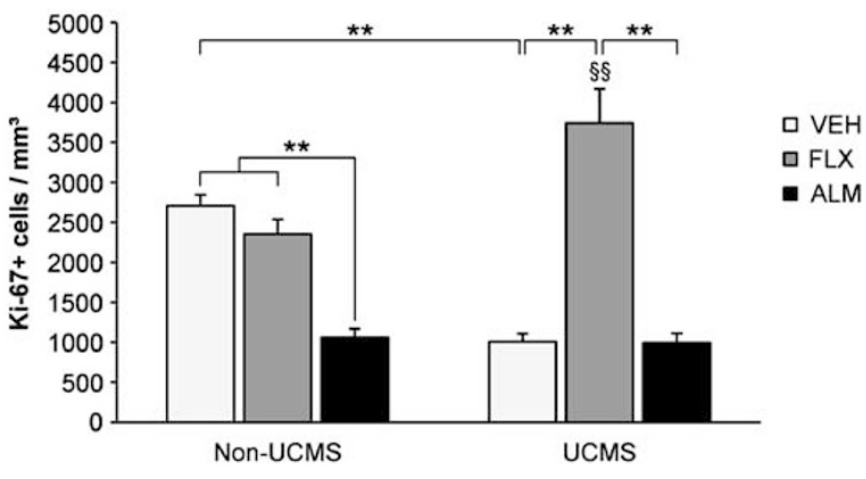

Ventral hippocampus

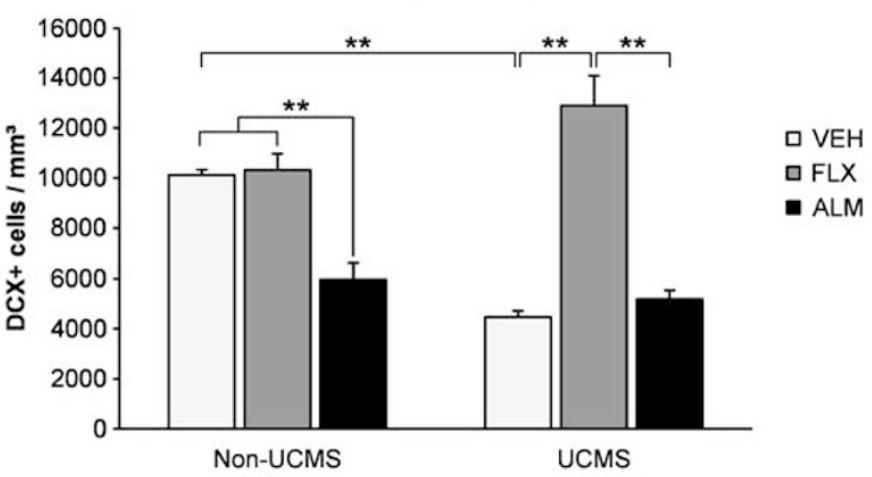

Ventral hippocampus

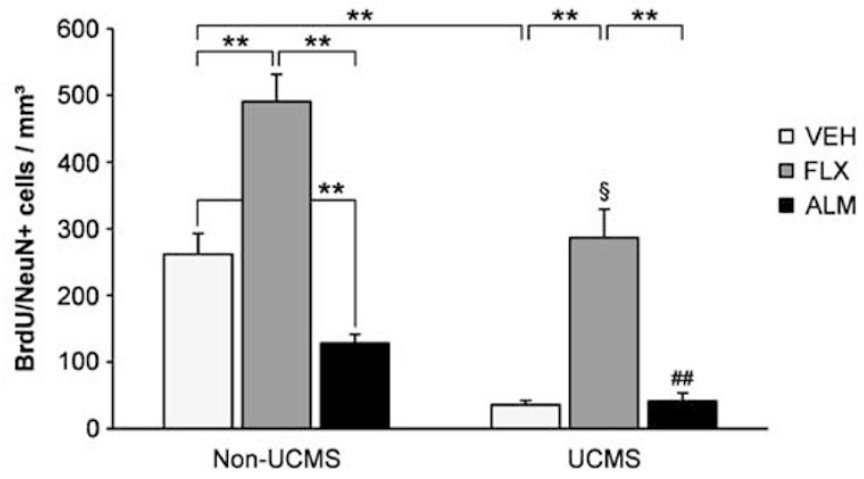




\section{DISCUSSION}

Since the first studies reporting on the putative link between orexins and $\mathrm{MD}$, there has been little progress in understanding the importance of such neuropeptidergic system in depressive-like and anxiety states. Indeed, preclinical and clinical studies dealing with the involvement of the orexinergic system in the onset of depressive symptoms led to conflicting results, ranging from a hypoactivity (Allard et al, 2004; Brundin et al, 2007a; Ito et al, 2008; Lutter et al, 2008; Taheri et al, 2001) or hyperactivity (Feng et al, 2007, 2008; Mikrouli et al, 2011; Salomon et al, 2003; von der Goltz et al, 2011) of orexinergic signaling. It has also been shown that intracerebroventricular administration or amygdala infusion of OX-A induces anxiety behaviors (Avolio et al, 2011; Suzuki et al, 2005). Furthermore, Johnson et al (2010) demonstrated that orexin neurons, especially in the DMH-PFA, are necessary for the occurrence of anxiety-like behavior in panic-prone rats since silencing hypothalamic $O X$ gene or intraperitoneal pretreatment with OXR1 antagonists blocks the panic responses. In line with the latter results, we recently reported that UCMS-subjected mice exhibited an increase of neuronal activation in the DMH-PFA orexinergic neurons compared with LH orexinergic neurons and that this effect was reversed by chronic FLX treatment (Nollet et al, 2011).

To the best of our knowledge, this study is the first to demonstrate a clear involvement of orexin signaling in a depressive-like state. One of our meaningful outcomes is the robust physical and behavioral antidepressant-like effects of the dual orexin receptor antagonist ALM. The UCMS procedure induced a depressive-like state in mice regarding their physical condition, with a clear deterioration of coat state that was reversed, as described previously, by chronic FLX treatment (Nollet et al, 2011; Santarelli et al, 2003; Surget et al, 2008, 2009), and more interestingly by chronic treatment with ALM. In addition, we found a regular increase of body weight during the entire protocol, while chronic ALM administration slackened the weight gain (without any significant differences in body weight when compared with corresponding VEH-treated groups). This reduction in weight growth could correspond to the decrease of food intake following the blockade of orexinergic system, as orexins are known to be involved in food-seeking (Cason et al, 2010; Thompson and Borgland, 2011). The behavioral data demonstrate that the UCMS regimen increased aggressiveness in the R-I test, despair behavior in the TST, and anxiety in the EPM and the NSF test. These behavioral changes were completely reversed by chronic treatment with FLX as demonstrated previously (Kurt et al, 2000; Nollet et al, 2011; Surget et al, 2008, 2009). ALM also reversed all the UCMS-induced behavioral changes besides the NSF test. In the latter behavioral test, the high latency to eat the pellet could reflect a reduced motivation to eat related to the anorexigenic effect of chronic antagonist injection. It is interesting to note that calorie restriction (corresponding to a $20-25 \%$ weight loss) is able to induce antidepressant-like effects (Lutter et al, 2008). Here, chronic ALM treatment did not induce weight loss but only a slight reduction of body weight gain, suggesting that antidepressant-like effects of ALM seen in our behavioral tests could not be attributed to calorie restriction or body weight loss. In addition, the ability of NSF test to reveal antidepressant-like effects can be influenced by the presence of hippocampal neurogenesis (David et al, 2009), which is highly reduced in our ALMtreated mice. Finally, in accordance with previous studies, chronic FLX and ALM treatments exerted an effect in nonUCMS animals in the TST (Kulkarni and Dhir, 2007; Nollet et al, 2011).

Since the restoration of the stress-induced HPA axis dysregulation is linked to the beneficial effects of antidepressant, we therefore assessed the effect of chronic ALM treatment on the putative UCMS-induced disruption of the HPA axis negative feedback loop through the DEX suppression test. Our findings show that chronic stress induced a dysregulation of HPA axis through the decrease of the response following the DEX suppression test. Indeed, both plasma corticosterone levels after DEX injection and Fos expression in parvocellular neurons of the PVN indicated a strong decrease of the negative feedback regulation of the HPA axis after UCMS. This negative feedback control was restored after FLX or ALM chronic treatments, suggesting that antidepressant effects obtained after both treatments could be linked to the restoration of a normal and functional negative feedback loop on HPA axis. These results corroborate the fact that, in most depressed patients with altered HPA axis negative feedback, remission and decreased risk of relapse are associated with the improvement of HPA axis functioning (Appelhof et al, 2006; Ising et al, 2007; Kunugi et al, 2006). Here, we have demonstrated that ALM restored the UCMS-induced HPA negative feedback disruption, sustaining the interaction between the PVN and the orexinergic neuronal population. Indeed, orexin neurons send direct projections to the PVN, particularly to the parvocellular cells (Yoshida et al, 2006), while neurons from the PVN innervate orexinergic neurons that express $\mathrm{CRH} 1$ and $\mathrm{CRH} 2$ receptors (Winsky-Sommerer et al, 2004). Moreover, OXR2 is abundant in the PVN (Marcus et al, 2001; Trivedi et al, 1998), and it has been shown that intracerebroventricular injection of selective OXR2 antagonist attenuates OX-A-induced increase of adrenocorticotropic hormone levels in rats (Chang et al, 2007). Consequently, the mechanism through which ALM restored the negative feedback on the HPA axis could be mediated by neurons located in the PVN through their orexin receptors 2 .

In this study, we found that chronic stress suppressed cell proliferation (Ki-67-expressing cells) and neurogenesis (DCX- and BrdU/NeuN-labeled cells) in the hippocampus, confirming previous reports (Czéh et al, 2001; Mineur et al, 2007; Pham et al, 2003). Furthermore, these suppressions affected more specifically the ventral part of the hippocampus, which strengthen evidences linking this region to stress and emotions (Fanselow and Dong, 2010). In accordance with what has been shown formerly, chronic FLX treatment reversed the UCMS-induced hippocampal cell proliferation and neurogenesis suppression (Malberg and Duman, 2003; Santarelli et al, 2003; Surget et al, 2008, 2011). Moreover, while no effect of FLX was observed in non-stressed mice regarding cell expressing immature neuronal marker DCX, these non-stressed animals treated with FLX exhibited a significant increase of mature neurons (4 weeks old) stained with BrdU/NeuN markers. This 
suggests that FLX treatment is able to increase survival or accelerate the maturation of new neurons in nonstressed animals (Perera et al, 2011; Wang et al, 2008), without increasing cell proliferation (Ki-67-labeled cells) or immature neurons density (DCX-expressing cells).

In our study, chronic treatment with the dual orexin receptor antagonist ALM neither did increase hippocampal cell proliferation nor neurogenesis. This result could be linked to the fact that, on the opposite, OX-A infusion increases cell proliferation (probably through the release of the neuropeptide $\mathrm{Y}$ ) without affecting the percentage of DCX-positive cells (Ito et al, 2008). This is critical as it has been shown that hippocampal neurogenesis is not required for the reversal of the behavioral and physical disruptions of UCMS by compounds targeting directly HPA stress axis response (CRH1 or V1b antagonists) (Surget et al, 2008). Moreover, $\mathrm{MCH}$ receptor 1 antagonist induces anxiolytic effect independently of hippocampal neurogenesis (David et al, 2007). Intriguingly, the blockade of orexinergic system even reduced cell proliferation and neurogenesis specifically in the ventral part of the hippocampus, without inducing depressive-like states per se, in line with several (but not all; Snyder et al, 2011) studies where no induced-depressivelike phenotypes have been observed in hippocampal neurogenesis-ablated mice (Santarelli et al, 2003; Surget et al, 2008, 2011). Considering that the ventral hippocampus is more related to emotional states and stress responses than its dorsal part, this result reinforces the idea that orexins play a central role in regulating emotional states and psychological stress (Furlong et al, 2009). Nevertheless, additional investigations will be necessary to fully understand this process.

Despite these new outcomes linking orexins and MD, several points need to be clarified by further studies. Here, the antidepressant-like effect underpinned by orexinergic system was obtained by blocking both orexin receptors, while a differential role of orexin receptors in depressivelike behavior has been underlined recently (Scott et al, 2011). Furthermore, although our findings suggest that ALM mostly induced an antidepressant-like effect by blocking orexinergic signaling on HPA axis, the therapeutic effect of orexinergic antagonist might also be the result of changes in sleep architecture (Li and Nattie, 2010) or interactions with serotoninergic system (Brown et al, 2001; Muraki et al, 2004). Finally, if our results are in line with previous studies showing a higher release of orexins as well as an increase of the number and activation of orexin neurons in several animal models of MD (Feng et al, 2007, 2008; Mikrouli et al, 2011; Nollet et al, 2011), they partially contradict other preclinical data in which genetic animal model of MD, chronic stress, or social defeat are associated with a decrease of orexinergic immunoreactivity and expression (Allard et al, 2004; Ito et al, 2009; Lutter et al, 2008; Taheri et al, 2001). The same discrepancies are observed in clinical studies in which depressive state have been linked to a decrease (Brundin et al, 2007a, 2007b, 2009; Rotter et al, 2011) or an increase (Palhagen et al, 2010; Salomon et al, 2003; von der Goltz et al, 2011) of OX-A level in cerebrospinal fluid or blood. It should also be noted that narcoleptic patients, characterized by low orexin levels, seem to present a higher rate of depression and anxiety (Dimitrova et al, 2011).
Taken together, our findings open new perspectives regarding the implication of the orexinergic system in MD. The fact that the dual orexin receptor antagonist ALM reinstates glucocorticoid feedback inhibition likely through the direct modulation of the PVN, the main central regulator of the stress axis, independently to the restoration of the hippocampal cell proliferation and neurogenesis strongly suggests that orexinergic system plays a significant role in depressive disorders, and underline the non-crucial role of hippocampal neurogenesis in the pathophysiology of MD.

\section{ACKNOWLEDGEMENTS}

We thank Dr François Jenck (Actelion Pharmaceuticals) and Actelion Pharmaceuticals for the gift of almorexant, Dr Petra $S$ van Nieuwenhuijzen (University of Sydney) for thoughtful comments on this article, as well as Anne-Marie Le Guisquet, Séverine Devers, Maryse Pingaud, Bruno Brizard, Lucette Garreau, Zuhal Gulhan, Jade Ezzedine, and Teddy Delavallée for assistance.

\section{DISCLOSURE}

The authors declare no conflict of interest.

\section{REFERENCES}

Aickin M, Gensler H (1996). Adjusting for multiple testing when reporting research results: the Bonferroni vs Holm methods. Am J Public Health 86: 726-728.

Airan RD, Meltzer LA, Roy M, Gong Y, Chen H, Deisseroth K (2007). High-speed imaging reveals neurophysiological links to behavior in an animal model of depression. Science 317: 819-823.

Allard JS, Tizabi Y, Shaffery JP, Trouth CO, Manaye K (2004). Stereological analysis of the hypothalamic hypocretin/orexin neurons in an animal model of depression. Neuropeptides 38: 311-315.

Alonso R, Griebel G, Pavone G, Stemmelin J, le Fur G, Soubrie P (2004). Blockade of $\mathrm{CRF}(1)$ or $\mathrm{V}(1 \mathrm{~b})$ receptors reverses stressinduced suppression of neurogenesis in a mouse model of depression. Mol Psychiatry 9: 278-286, 224.

Appelhof BC, Huyser J, Verweij M, Brouwer JP, van Dyck R, Fliers $\mathrm{E}$ et al (2006). Glucocorticoids and relapse of major depression (dexamethasone/corticotropin-releasing hormone test in relation to relapse of major depression). Biol Psychiatry 59: 696-701. Aston-Jones G, Smith RJ, Sartor GC, Moorman DE, Massi L, Tahsili-Fahadan $\mathrm{P}$ et al (2010). Lateral hypothalamic orexin/ hypocretin neurons: a role in reward-seeking and addiction. Brain Res 1314: 74-90.

Avolio E, Alo R, Carelli A, Canonaco M (2011). Amygdalar orexinergic-GABAergic interactions regulate anxiety behaviors of the Syrian golden hamster. Behav Brain Res 218: 288-295.

Brisbare-Roch C, Dingemanse J, Koberstein R, Hoever P, Aissaoui $\mathrm{H}$, Flores $\mathrm{S}$ et al (2007). Promotion of sleep by targeting the orexin system in rats, dogs and humans. Nat Med 13: 150-155.

Brown RE, Sergeeva O, Eriksson KS, Haas HL (2001). Orexin A excites serotonergic neurons in the dorsal raphe nucleus of the rat. Neuropharmacology 40: 457-459.

Brundin L, Bjorkqvist M, Petersen A, Traskman-Bendz L (2007a). Reduced orexin levels in the cerebrospinal fluid of suicidal patients with major depressive disorder. Eur Neuropsychopharmacol 17: 573-579. 
Brundin L, Bjorkqvist M, Traskman-Bendz L, Petersen A (2009). Increased orexin levels in the cerebrospinal fluid the first year after a suicide attempt. J Affect Disord 113: 179-182.

Brundin L, Petersen A, Bjorkqvist M, Traskman-Bendz L (2007b). Orexin and psychiatric symptoms in suicide attempters. J Affect Disord 100: 259-263.

Cao M, Guilleminault C (2011). Hypocretin and its emerging role as a target for treatment of sleep disorders. Curr Neurol Neurosci Rep 11: 227-234.

Cason AM, Smith RJ, Tahsili-Fahadan P, Moorman DE, Sartor GC, Aston-Jones G (2010). Role of orexin/hypocretin in rewardseeking and addiction: implications for obesity. Physiol Behav 100: 419-428.

Chang H, Saito T, Ohiwa N, Tateoka M, Deocaris CC, Fujikawa T et al (2007). Inhibitory effects of an orexin-2 receptor antagonist on orexin A- and stress-induced ACTH responses in conscious rats. Neurosci Res 57: 462-466.

Cryan JF, Holmes A (2005). The ascent of mouse: advances in modelling human depression and anxiety. Nat Rev Drug Discov 4: 775-790.

Czéh B, Michaelis T, Watanabe T, Frahm J, de Biurrun G, van Kampen $M$ et al (2001). Stress-induced changes in cerebral metabolites, hippocampal volume, and cell proliferation are prevented by antidepressant treatment with tianeptine. Proc Natl Acad Sci USA 98: 12796-12801.

David DJ, Klemenhagen KC, Holick KA, Saxe MD, Mendez I, Santarelli L et al (2007). Efficacy of the MCHR1 antagonist $\mathrm{N}$-[3(1-\{[4-(3,4-difluorophenoxy)phenyl]methyl\}(4-piperidyl))-4methylphen yl]-2-methylpropanamide (SNAP 94847) in mouse models of anxiety and depression following acute and chronic administration is independent of hippocampal neurogenesis. J Pharmacol Exp Ther 321: 237-248.

David DJ, Samuels BA, Rainer Q, Wang JW, Marsteller D, Mendez I et al (2009). Neurogenesis-dependent and -independent effects of fluoxetine in an animal model of anxiety/depression. Neuron 62: 479-493.

de Lecea L, Kilduff TS, Peyron C, Gao X, Foye PE, Danielson PE et al (1998). The hypocretins: hypothalamus-specific peptides with neuroexcitatory activity. Proc Natl Acad Sci USA 95: 322-327.

Dimitrova A, Fronczek R, Van der Ploeg J, Scammell T, Gautam S, Pascual-Leone A et al (2011). Reward-seeking behavior in human narcolepsy. J Clin Sleep Med 7: 293-300.

El Hage W, Powell JF, Surguladze SA (2009). Vulnerability to depression: what is the role of stress genes in gene $\times$ environment interaction? Psychol Med 39: 1407-1411.

Fanselow MS, Dong HW (2010). Are the dorsal and ventral hippocampus functionally distinct structures? Neuron 65: 7-19.

Feng P, Vurbic D, Wu Z, Hu Y, Strohl KP (2008). Changes in brain orexin levels in a rat model of depression induced by neonatal administration of clomipramine. J Psychopharmacol 22: 784-791.

Feng P, Vurbic D, Wu Z, Strohl KP (2007). Brain orexins and wake regulation in rats exposed to maternal deprivation. Brain Res 1154: $163-172$.

Franklin KBJ, Paxinos G (2008). The Mouse Brain in Stereotaxic Coordinates 3rd edn Academic Press, Elsevier: New York.

Furlong TM, Vianna DM, Liu L, Carrive P (2009). Hypocretin/ orexin contributes to the expression of some but not all forms of stress and arousal. Eur J Neurosci 30: 1603-1614.

Harris GC, Wimmer M, Aston-Jones G (2005). A role for lateral hypothalamic orexin neurons in reward seeking. Nature 437: 556-559.

Herman JP, Ostrander MM, Mueller NK, Figueiredo H (2005). Limbic system mechanisms of stress regulation: hypothalamopituitary-adrenocortical axis. Prog Neuropsychopharmacol Biol Psychiatry 29: 1201-1213.
Ising M, Horstmann S, Kloiber S, Lucae S, Binder EB, Kern N et al (2007). Combined dexamethasone/corticotropin releasing hormone test predicts treatment response in major depressiona potential biomarker? Biol Psychiatry 62: 47-54.

Ito N, Yabe T, Gamo Y, Nagai T, Oikawa T, Yamada H et al (2008). I.c.v. administration of orexin-A induces an antidepressive-like effect through hippocampal cell proliferation. Neuroscience 157: 720-732.

Ito N, Yabe T, Nagai T, Oikawa T, Yamada H, Hanawa T (2009). A possible mechanism underlying an antidepressive-like effect of Kososan, a Kampo medicine, via the hypothalamic orexinergic system in the stress-induced depression-like model mice. Biol Pharm Bull 32: 1716-1722.

Jiang W, Zhang Y, Xiao L, van Cleemput J, Ji SP, Bai G et al (2005). Cannabinoids promote embryonic and adult hippocampus neurogenesis and produce anxiolytic- and antidepressant-like effects. J Clin Invest 115: 3104-3116.

Johnson PL, Truitt W, Fitz SD, Minick PE, Dietrich A, Sanghani S et al (2010). A key role for orexin in panic anxiety. Nat Med 16: $111-115$.

Kang JE, Lim MM, Bateman RJ, Lee JJ, Smyth LP, Cirrito JR et al (2009). Amyloid-beta dynamics are regulated by orexin and the sleep-wake cycle. Science 326: 1005-1007.

Keller MC, Neale MC, Kendler KS (2007). Association of different adverse life events with distinct patterns of depressive symptoms. Am J Psychiatry 164: 1521-1529.

Kulkarni SK, Dhir A (2007). Effect of various classes of antidepressants in behavioral paradigms of despair. Prog Neuropsychopharmacol Biol Psychiatry 31: 1248-1254.

Kunugi H, Ida I, Owashi T, Kimura M, Inoue Y, Nakagawa S et al (2006). Assessment of the dexamethasone/CRH test as a statedependent marker for hypothalamic-pituitary-adrenal (HPA) axis abnormalities in major depressive episode: a Multicenter Study. Neuropsychopharmacology 31: 212-220.

Kurt M, Arik AC, Celik S (2000). The effects of sertraline and fluoxetine on anxiety in the elevated plus-maze test in mice. $J$ Basic Clin Physiol Pharmacol 11: 173-180.

Li A, Nattie E (2010). Antagonism of rat orexin receptors by almorexant attenuates central chemoreception in wakefulness in the active period of the diurnal cycle. J Physiol 588: 2935-2944.

Lopez M, Tena-Sempere M, Dieguez C (2010). Cross-talk between orexins (hypocretins) and the neuroendocrine axes (hypothalamic-pituitary axes). Front Neuroendocrinol 31: 113-127.

Lutter M, Krishnan V, Russo SJ, Jung S, McClung CA, Nestler EJ (2008). Orexin signaling mediates the antidepressant-like effect of calorie restriction. J Neurosci 28: 3071-3075.

Malberg JE, Duman RS (2003). Cell proliferation in adult hippocampus is decreased by inescapable stress: reversal by fluoxetine treatment. Neuropsychopharmacology 28: $1562-1571$.

Malberg JE, Eisch AJ, Nestler EJ, Duman RS (2000). Chronic antidepressant treatment increases neurogenesis in adult rat hippocampus. J Neurosci 20: 9104-9110.

Marcus JN, Aschkenasi CJ, Lee CE, Chemelli RM, Saper CB, Yanagisawa $M$ et al (2001). Differential expression of orexin receptors 1 and 2 in the rat brain. J Comp Neurol 435: 6-25.

McEwen BS, Magarinos AM, Reagan LP (2002). Structural plasticity and tianeptine: cellular and molecular targets. Eur Psychiatry 17(Suppl 3): 318-330.

Mikrouli E, Wortwein G, Soylu R, Mathe AA, Petersen S (2011). Increased numbers of orexin/hypocretin neurons in a genetic rat depression model. Neuropeptides 45: 401-406.

Mineur YS, Belzung C, Crusio WE (2007). Functional implications of decreases in neurogenesis following chronic mild stress in mice. Neuroscience 150: 251-259. 
Mineur YS, Prasol DJ, Belzung C, Crusio WE (2003). Agonistic behavior and unpredictable chronic mild stress in mice. Behav Genet 33: 513-519.

Mizoguchi K, Ishige A, Aburada M, Tabira T (2003). Chronic stress attenuates glucocorticoid negative feedback: involvement of the prefrontal cortex and hippocampus. Neuroscience 119: 887-897.

Muraki Y, Yamanaka A, Tsujino N, Kilduff TS, Goto K, Sakurai T (2004). Serotonergic regulation of the orexin/hypocretin neurons through the 5-HT1A receptor. J Neurosci 24: 7159-7166.

Nestler EJ, Barrot M, DiLeone RJ, Eisch AJ, Gold SJ, Monteggia LM (2002). Neurobiology of depression. Neuron 34: 13-25.

Nollet M, Gaillard P, Minier F, Tanti A, Belzung C, Leman S (2011). Activation of orexin neurons in dorsomedial/perifornical hypothalamus and antidepressant reversal in a rodent model of depression. Neuropharmacology 61: 336-346.

Palhagen S, Qi H, Martensson B, Walinder J, Granerus AK, Svenningsson P (2010). Monoamines, BDNF, IL-6 and corticosterone in CSF in patients with Parkinson's disease and major depression. J Neurol 257: 524-532.

Perera TD, Dwork AJ, Keegan KA, Thirumangalakudi L, Lipira $\mathrm{CM}$, Joyce $\mathrm{N}$ et al (2011). Necessity of hippocampal neurogenesis for the therapeutic action of antidepressants in adult nonhuman primates. PLoS One 6: e17600.

Peyron C, Tighe DK, van den Pol AN, de Lecea L, Heller HC, Sutcliffe JG et al (1998). Neurons containing hypocretin (orexin) project to multiple neuronal systems. J Neurosci 18: 9996-10015.

Pham K, Nacher J, Hof PR, McEwen BS (2003). Repeated restraint stress suppresses neurogenesis and induces biphasic PSA-NCAM expression in the adult rat dentate gyrus. Eur J Neurosci 17: 879-886.

Revesz D, Tjernstrom M, Ben-Menachem E, Thorlin T (2008). Effects of vagus nerve stimulation on rat hippocampal progenitor proliferation. Exp Neurol 214: 259-265.

Rotter A, Asemann R, Decker A, Kornhuber J, Biermann T (2011). Orexin expression and promoter-methylation in peripheral blood of patients suffering from major depressive disorder. J Affect Disord 131: 186-192.

Sakurai T, Amemiya A, Ishii M, Matsuzaki I, Chemelli RM, Tanaka $\mathrm{H}$ et al (1998). Orexins and orexin receptors: a family of hypothalamic neuropeptides and $G$ protein-coupled receptors that regulate feeding behavior. Cell 92: 573-585.

Sakurai T, Mieda M, Tsujino N (2010). The orexin system: roles in sleep/wake regulation. Ann NY Acad Sci 1200: 149-161.

Salomon RM, Ripley B, Kennedy JS, Johnson B, Schmidt D, Zeitzer JM et al (2003). Diurnal variation of cerebrospinal fluid hypocretin-1 (Orexin-A) levels in control and depressed subjects. Biol Psychiatry 54: 96-104.

Santarelli L, Saxe M, Gross C, Surget A, Battaglia F, Dulawa S et al (2003). Requirement of hippocampal neurogenesis for the behavioral effects of antidepressants. Science 301: 805-809.

Scott MM, Marcus JN, Pettersen A, Birnbaum SG, Mochizuki T, Scammell TE et al (2011). Hcrtr1 and 2 signaling differentially regulates depression-like behaviors. Behav Brain Res 222: 289-294.

Sibille E, Wang Y, Joeyen-Waldorf J, Gaiteri C, Surget A, Oh S et al (2009). A molecular signature of depression in the amygdala. Am J Psychiatry 166: 1011-1024.
Snyder JS, Soumier A, Brewer M, Pickel J, Cameron HA (2011). Adult hippocampal neurogenesis buffers stress responses and depressive behaviour. Nature 476: 458-461.

Spinazzi R, Andreis PG, Rossi GP, Nussdorfer GG (2006). Orexins in the regulation of the hypothalamic-pituitary-adrenal axis. Pharmacol Rev 58: 46-57.

Steru L, Chermat R, Thierry B, Simon P (1985). The tail suspension test: a new method for screening antidepressants in mice. Psychopharmacology (Berl) 85: 367-370.

Surget A, Saxe M, Leman S, Ibarguen-Vargas Y, Chalon S, Griebel $\mathrm{G}$ et al (2008). Drug-dependent requirement of hippocampal neurogenesis in a model of depression and of antidepressant reversal. Biol Psychiatry 64: 293-301.

Surget A, Tanti A, Leonardo ED, Laugeray A, Rainer Q, Touma C et al (2011). Antidepressants recruit new neurons to improve stress response regulation. Mol Psychiatry 16: 1177-1188.

Surget A, Wang Y, Leman S, Ibarguen-Vargas Y, Edgar N, Griebel $\mathrm{G}$ et al (2009). Corticolimbic transcriptome changes are statedependent and region-specific in a rodent model of depression and of antidepressant reversal. Neuropsychopharmacology 34: $1363-1380$

Suzuki M, Beuckmann CT, Shikata K, Ogura H, Sawai T (2005). Orexin-A (hypocretin-1) is possibly involved in generation of anxiety-like behavior. Brain Res 1044: 116-121.

Taheri S, Gardiner J, Hafizi S, Murphy K, Dakin C, Seal L et al (2001). Orexin A immunoreactivity and preproorexin mRNA in the brain of Zucker and WKY rats. NeuroReport 12: 459-464.

Tanti A, Belzung C (2010). Open questions in current models of antidepressant action. Br J Pharmacol 159: 1187-1200.

Thompson JL, Borgland SL (2011). A role for hypocretin/orexin in motivation. Behav Brain Res 217: 446-453.

Trivedi P, Yu H, MacNeil DJ, van der Ploeg LH, Guan XM (1998). Distribution of orexin receptor mRNA in the rat brain. FEBS Lett 438: 71-75.

Ulrich-Lai YM, Herman JP (2009). Neural regulation of endocrine and autonomic stress responses. Nat Rev Neurosci 10: 397-409.

von der Goltz C, Koopmann A, Dinter C, Richter A, Grosshans M, Fink $\mathrm{T}$ et al (2011). Involvement of orexin in the regulation of stress, depression and reward in alcohol dependence. Horm Behav 60: 644-650.

Wang JW, David DJ, Monckton JE, Battaglia F, Hen R (2008). Chronic fluoxetine stimulates maturation and synaptic plasticity of adult-born hippocampal granule cells. J Neurosci 28: 1374-1384.

Willner P (1997). Validity, reliability and utility of the chronic mild stress model of depression: a 10-year review and evaluation. Psychopharmacology (Berl) 134: 319-329.

Winsky-Sommerer R, Yamanaka A, Diano S, Borok E, Roberts AJ, Sakurai $\mathrm{T}$ et al (2004). Interaction between the corticotropinreleasing factor system and hypocretins (orexins): a novel circuit mediating stress response. J Neurosci 24: 11439-11448.

Yoshida K, McCormack S, Espana RA, Crocker A, Scammell TE (2006). Afferents to the orexin neurons of the rat brain. J Comp Neurol 494: 845-861.

Yoshimizu T, Chaki S (2004). Increased cell proliferation in the adult mouse hippocampus following chronic administration of group II metabotropic glutamate receptor antagonist, MGS0039. Biochem Biophys Res Commun 315: 493-496.

Supplementary Information accompanies the paper on the Neuropsychopharmacology website (http://www.nature.com/npp) 